\title{
Group Velocity Dispersion Reduction in Fibre-based Slow-light Systems via Stimulated Brillouin Scattering
}

Ronny Henker

Deutsche Telekom Fachhochschule Leipzig

A. Wiatrek

Deutsche Telekom Fachhochschule Leipzig

K. U. Lauterbach

Deutsche Telekom Fachhochschule Leipzig

See next page for additional authors

Follow this and additional works at: https://arrow.tudublin.ie/engscheceart

Part of the Electrical and Computer Engineering Commons

\section{Recommended Citation}

Henker, R. et al. (2008) Group velocity dispersion reduction in fibre-based slow-light systems via stimulated Brillouin scattering. Electronics Letters,September 25, 2008, Vol.44, pp.1185-1186. doi:10.1049/el:20082007.

This Article is brought to you for free and open access by the School of Electrical and Electronic Engineering at ARROW@TU Dublin. It has been accepted for inclusion in Articles by an authorized administrator of ARROW@TU Dublin. For more information, please contact arrow.admin@tudublin.ie, aisling.coyne@tudublin.ie, gerard.connolly@tudublin.ie.

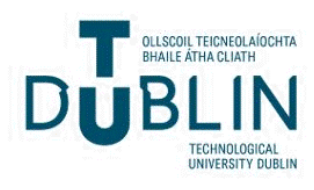




\section{Authors}

Ronny Henker, A. Wiatrek, K. U. Lauterbach, Max Ammann, Andreas Schwarzbacher, and Thomas Schneider 


\section{Group velocity dispersion reduction in fibre-based slow-light systems via stimulated Brillouin scattering}

R. Henker, A. Wiatrek, K.-U. Lauterbach, M.J. Ammann, A.T. Schwarzbacher and T. Schneider

Presented are theoretical investigations and simulations of a reduction of the group velocity dispersion (GVD) in slow-light systems by an optimisation of the gain spectrum of the stimulated Brillouin scattering (SBS). A comparison between a natural Brillouin gain spectrum and a broadened Brillouin gain spectrum with and without two additional losses at its wings is demonstrated. The focus of the theoretical examination was set on the superposition of a gain and two losses with an equal magnitude. Therefore, a low GVD and a high group index change could be achieved and hence, a small pulse distortion and a high time delay can be expected.

Introduction: Different slow-light systems have been discussed and developed as a powerful tool to reduce the group velocity of optical signals during the last years. This area has enjoyed much recent interest because of the potential practical applications in optical telecommunication and information systems like optical buffers, synchronisers or equalisers.

In particular, the nonlinear effect of stimulated Brillouin scattering (SBS) has become important for fibre-based slow-light systems [1, 2], because it offers several advantages compared to other methods. But, it also has two disadvantages. First, the maximum time delay is limited owing to the saturation of the Brillouin amplification process and, secondly, the delay of the pulses is accompanied by pulse distortions (pulse shape broadening) owing to the group velocity dispersion (GVD) and the spectral narrowing by the SBS gain. Therefore, different mechanisms for tailoring the shape of the Brillouin spectrum have been investigated. By a broadening of the Brillouin gain pump source via multiple pump lines [3] or a direct modulation with a noise source $[4,5]$, a uniform spectrum over a broad bandwidth can be produced. Hence, the pulse distortions can be reduced [6] and higher delayable data rates are possible. However, this decreases the time delay significantly. An enhancement of the maximum time delay can be achieved by a superposition of Brillouin gain and loss spectra, which decouples the time delay from the amplifier gain [7]. In [8] we have already presented a mechanism to increase the maximum time delay drastically to four times the initial pulse width. In contrast to the studied techniques with multiple gain lines in [3] it is based on a superposition of one Brillouin gain spectrum with two additional Brillouin loss spectra at its wings.

In this Letter, we present the theory and simulations that this method has also the potential to reduce the GVD and the pulse distortions drastically.

Theory: The propagation of a pulse in a medium as a function of the frequency $\omega$ can be described by the complex wave number [9]:

$$
k(\omega)=\sum_{i=0}^{\infty} \frac{k_{i}\left(\omega-\omega_{0}\right)^{i}}{i !} \text { with } k_{i}=\left[\frac{d^{i} k}{d \omega^{i}}\right]_{\omega=\omega_{0}}
$$

The first derivative $k_{1}=d k / d \omega$ corresponds to the reciprocal group velocity whereas the second derivative $k_{2}=d^{2} k / d \omega^{2}$ is the GVD. For a Brillouin gain with a Lorentzian shape the complex wave number can be written as [9]:

$$
k(\omega)=\frac{n_{0}}{c} \omega+\frac{g_{1}}{z}\left(\frac{\gamma_{1}}{\left(\omega-\omega_{0}\right)+j \gamma_{1}}\right)
$$

where $z$ is the length of the medium, $c$ is the speed of light in vacuum, $n_{0}$ is the refractive index, $\omega_{0}$ is the line centre of the gain distribution, $\gamma_{1}$ is the half FWHM-bandwidth of the SBS gain as angular frequency and $g_{1}=g P L_{e f f} / A_{\text {eff }}$ is the line centre gain with $g$ as the Brillouin coefficient, $P$ as input pump power, and $L_{\text {eff }}$ and $A_{\text {eff }}$ as the effective length and area of the fibre. The imaginary part of (2) leads to an amplification of the pulses and the real part to a phase change. Furthermore, the derivation of the real part leads to the group index change, which causes the slow-light time delay. In the line centre it is $\Delta t=g_{1} / \gamma_{1}$. This delay is accompanied with a broadening of the output pulse due to the GVD and higher-order terms of (1). The pulse broadening factor $B$ as the relation between the output $\tau_{\text {out }}$ and input pulse width $\tau_{\text {in }}$ can be described by [6]

$$
B=\frac{\tau_{\text {out }}}{\tau_{\text {in }}}=\sqrt{1+\frac{16 \ln 2}{\tau_{\text {in }}^{2} \gamma_{1}^{2}} g_{1}}
$$

As can be seen from (3) the broadening factor decreases for high gain bandwidths. Hence, the pulse distortions can be reduced by a gain broadening. If the Brillouin gain spectrum is broadened it becomes Gaussian shaped and the complex wave number changes to [5]:

$$
k(\omega)=\frac{n_{0}}{c} \omega+\frac{g_{1}}{j z}\left(e^{-\left(\frac{\omega-\omega_{0}}{\gamma_{G}}\right)^{2}} \operatorname{erfc}\left(-j \frac{\omega-\omega_{0}}{\gamma_{G}}\right)\right)
$$

with $\gamma_{G}$ as the $1 / e$-bandwidth and erfc () as the complementary error function. Then, the time delay is decreased and can be written as $\Delta t=2 g_{1} /\left(\gamma_{G} \sqrt{ } \pi\right)$. But, if the value of the Brillouin gain broadening is comparable with the Brillouin shift, the influence of the Brillouin loss leads to an enhancement of the time delay at the centre of the Stokes resonance [5], which is not further investigated here. However, by adding two narrow Lorentzian shaped Brillouin loss spectra $g_{2}$, with a separation $2 \delta$ and a half FWHM-bandwidth $\gamma_{2}$, at the wings of the gain the time delay can be enhanced to $\Delta t=2 g_{1} /\left(\gamma_{G} \sqrt{ } \pi\right)+$ $\left(\delta^{2}-\gamma_{2}^{2}\right) /\left(\delta^{2}+\gamma_{2}^{2}\right)^{2}$ and (2) and (4) have to be expanded by the term $[8]:$

$$
\ldots-\frac{g_{2}}{z}\left(\frac{\gamma_{2}}{\left(\omega-\left(\omega_{0} \pm \delta\right)\right)+j \gamma_{2}}\right)
$$

The inset of Fig. 1 shows the superposition of a Brillouin gain spectrum with two losses at its wings. The gain bandwidth, the time delay and the GVD can be adjusted and controlled by the separation of the losses $2 \delta$.

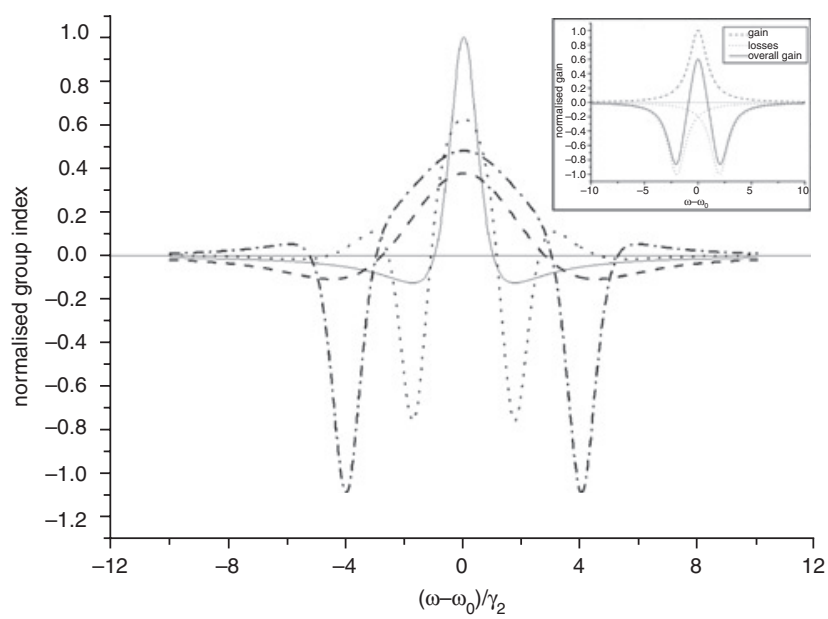

Fig. 1 Normalised group index for natural gain (solid) and three times broadened gain (dashed) with losses at $\delta= \pm 1.7 \gamma_{2}$ (dotted) and $\delta=$ $\pm 4 \gamma_{2}$ (dashed-dotted)

Inset shows the superposition (solid) of Brillouin gain (dashed) with two losses (dotted) at its wings

Simulation results: The simulation results for equal gain and losses $g_{1}=g_{2}$ are shown in Figs. 1 and 2. The group index was normalised to the maximum value of the natural gain case. As can be seen, a high group index and hence a high time delay in the line centre is achieved with a normal gain (solid line). But, the GVD is also very high for this case and the pulses will experience a huge distortion. A much higher group index could be achieved by adding two loss spectra at the wings of the normal gain [8], which is not examined here because there is no improvement of the GVD. But, if the gain is broadened (here $\gamma_{G}=3 \gamma_{2}$, dashed line), the GVD is reduced drastically and it runs linearly with a small slope in the middle of the Brillouin profile. However, the group index in the line centre of the distribution is decreased as well and it is barely $38 \%$, as for the normal gain. 


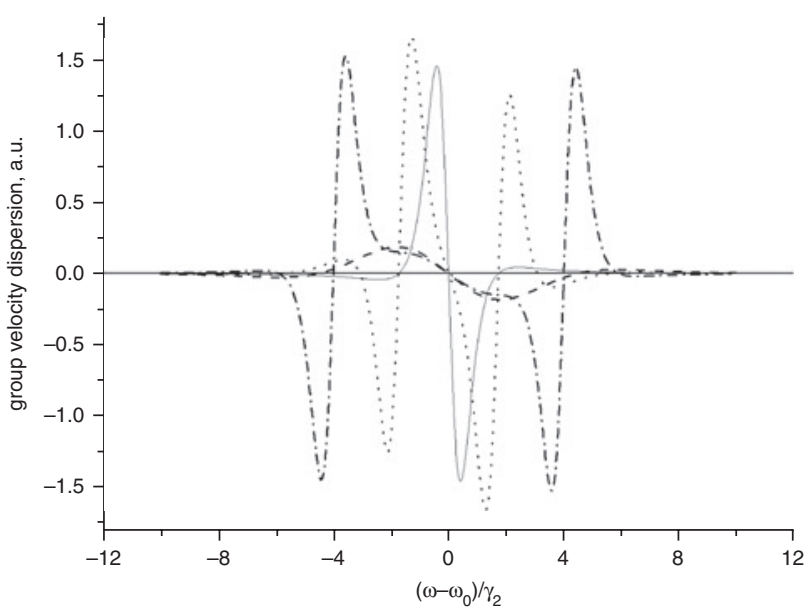

Fig. 2 Group velocity dispersion for natural gain (solid) and three times broadened gain (dashed) with losses at $\delta= \pm 1.7 \gamma_{2}$ (dotted) and $\delta= \pm 4 \gamma_{2}$ (dashed-dotted)

With the additional losses at the wings of the broadened gain the time delay can be enhanced. Thereby, the separation of the two losses influences the height of the group index and the slope of the GVD directly. If the losses are far away from the line centre their impact is negligible. But, with a smaller separation the gain bandwidth is constrained again. By varying $\delta$ we determined two optimal operating points for the separation. At $\delta= \pm 4 \gamma_{2}$ (dashed-dotted line) the maximum bandwidth is achieved. In this case, the group index in the line centre is nearly $50 \%$, as for a normal gain, whereas the GVD shows the same progress as for the broadened gain without losses. Hence, the pulse distortions should be at the minimum.

The highest group index for this case can be achieved by a loss separation of $\delta= \pm 1.7 \gamma_{2}$ (dotted line). Then, the group index is actually $63 \%$. But, this is at the expense of the GVD, which runs with an even steeper slope in the middle of the Brillouin profile. However, the slope is still smoother than for the normal gain case.

With a very broad and flat Brillouin spectrum the GVD could even be reduced to zero within the flat gain region around the line centre. If the spectral components of the pulse do not exceed this region it would not experience distortions due to the GVD. However, the time delay would be very low and there is still a large change of the GVD at the edges of the Brillouin spectrum, which leads to distortions of the pulse with an increased bandwidth. On the other hand this can be used to achieve zero-pulse-broadening by a compression of the pulse, which compensates for the broadening owing to the spectral narrowing by the SBS gain.

Conclusions: We have shown that a superposition of a Brillouin gain with two losses at its wings has the potential to reduce the GVD drastically in fibre-based slow-light systems. As an example the gain was broadened by a factor of three and the separation of the losses was adjusted to achieve the maximum bandwidth or the maximum time delay. Hence, the pulse distortions can be decreased in addition to an enhancement of the time delay. However, this needs further investigations.

Acknowledgments: We gratefully acknowledge the technical help of M. Junker and J. Klinger from HfT Leipzig. K.-U. Lauterbach and R. Henker acknowledge the financial support from Deutsche Telekom.

(C) The Institution of Engineering and Technology 2008

10 July 2008

Electronics Letters online no: 20082007

doi: 10.1049/el:20082007

R. Henker, A. Wiatrek, K.-U. Lauterbach and T. Schneider (Hochschule für Telekommunikation Leipzig (FH), Gustav-Freytag-Str. 43-45, Leipzig 04277, Germany)

E-mail: ronny.henker@hft-leipzig.de

R. Henker, A. Wiatrek, K.-U. Lauterbach, M.J. Ammann and A.T. Schwarzbacher (School of Electronic \& Communications Engineering, Dublin Institute of Technology, Kevin Street, Dublin 8, Ireland)

\section{References}

1 Song, K., González-Herráez, M., and Thévenaz, L.: 'Observation of pulse delaying and advancement in optical fibres using stimulated Brillouin scattering', Opt. Express, 2005, 13, (1), pp. 82-88

2 Okawachi, Y., Bigelow, M.S.M., Sharping, J.E., Zhu, Z., Schweinsberg, A., Gauthier, D.J., Boyd, R.W., and Gaeta, A.L.: 'Tunable all-optical delays via Brillouin slow light in an optical fiber', Phys. Rev. Lett., 2005, 94, (15), p. 153902-1-153902-4

3 Shi, Z., Pant, R., Zhu, Z., Stenner, M.D., Neifeld, M.A., Gauthier, D.J., and Boyd, R.W.: 'Design of a tunable time-delay element using multiple gain lines for increased fractional delay with high data fidelity', Opt. Lett., 2007, 32, (14), pp. 1986-1988

4 González-Herráez, M., Song, K., and Thévenaz, L.: 'Arbitrarybandwidth Brillouin slow light in optical fibres', Opt. Express, 2006, 14, (4), pp. 1395-1400

5 Zhu, Z., Dawes, A.M.C., Gauthier, D.J., Zhang, L., and Willner, A.E.: 'Broadband SBS slow light in an optical fiber', J. Lightwave Technol., 2007, 25, (1), pp. 201-206

6 Schneider, T., Henker, R., Lauterbach, K.-U., and Junker, M.: 'Distortion reduction in slow light systems based on stimulated Brillouin scattering', Opt. Express, 2008, 16, (11), pp. 8280-8285

7 Chin, S., Gonzalez-Herraez, M., and Thévenaz, L.: 'Zero-gain slow \& fast light propagation in an optical fiber', Opt. Express, 2006, 14, (22), pp. 10684-10692

8 Schneider, T., Henker, R., Lauterbach, K.-U., and Junker, M.: 'Comparison of delay enhancement mechanisms for SBS-based slow light systems', Opt. Express, 2007, 15, (15), pp. 9606-9613

9 Stenner, M.D., Neifeld, M.A., Zhu, Z., Dawes, A.M.C., and Gauthier, D.J.: 'Distortion management in slow-light pulse delay', Opt. Express, 2005, 13, (25), pp. 9995-10002 